\title{
Effects of Tribulus terrestris on monosodium iodoacetate-induced osteoarthritis pain in rats
}

\author{
YOUNG JIN PARK $^{1 *}$, YOUNG-RAK CHO ${ }^{1 *}$, JOA SUB OH ${ }^{2}$ and EUN-KYUNG AHN ${ }^{1}$ \\ ${ }^{1}$ Bio-center, Gyeonggi Institute of Science and Technology Promotion, Suwon, Gyeonggi 443-270; ${ }^{2}$ Department of \\ Pharmacy, College of Pharmacy, Dankook University, Cheonan, Chungcheongnam 330-714, Republic of Korea
}

Received July 16, 2016; Accepted February 23, 2017

DOI: $10.3892 / \mathrm{mmr} .2017 .7296$

\begin{abstract}
Tribulus terrestris L. (T. terrestris) has been used as a traditional medicine for the treatment of diuretic, lithontriptic, edema and urinary infections. Previous studies have indicated that it is effective in improving inflammation by regulating tumor necrosis factor- $\alpha$ (TNF)- $\alpha$, interleukin (IL)-6, IL-10, nitric oxide (NO) and cyclooxygenase (COX)-2. However, the effects and mechanism of action of T. terrestris on osteoarthritis $(\mathrm{OA})$ remain unknown. Therefore, the present study aimed to evaluate the effects of the ethanolic extract of $T$. terrestris (ETT) in a monosodium iodoacetate (MIA)-induced OA animal model. OA was induced in LEW/SSNHSD rats by intra-articular injection of MIA. Morphometric changes and parameters of the tibial trabecular bone were determined using micro-computed tomography. The molecular mechanisms of ETT in OA were investigated using reverse transcription-polymerase chain reaction, western blotting and gelatin zymogram analysis. Treatment with ETT attenuated MIA-induced OA, and this effect was mediated by the downregulation of NO synthase 2, COX-2, TNF- $\alpha$ and IL-6. Furthermore, the ETT-mediated attenuation of OA was also
\end{abstract}

Correspondence to: Dr Eun-Kyung Ahn, Bio-center, Gyeonggi Institute of Science and Technology Promotion, 147 Gwanggyo-ro, Suwon, Gyeonggi 443-270, Republic of Korea

E-mail: aek@gstep.re.kr

${ }^{*}$ Contributed equally

Abbreviations: BS/BV, bone surface area/bone volume; BV/TV, bone volume fraction; COX, cyclooxygenase; ERK, extracellular signal-regulated kinase; ETT, ethanolic extract of Tribulus terrestris; IL, interleukin; MIA, monosodium iodoacetate; micro-CT, micro-computed tomography; MMPs, matrix metalloproteinases; NOS, nitric oxide synthase; NSAIDs, nonsteroidal anti-inflammatory drugs; OA, osteoarthritis; RT-PCR, reverse transcription-polymerase chain reaction; Tb.Sp, trabecular spacing; Tb.Th, trabecular thickness; TNF- $\alpha$, tumor necrosis factor- $\alpha$

Key words: Tribulus terrestris L., osteoarthritis, monosodium iodoacetate, cyclooxygenase-2, matrix metalloproteinases dependent on the expression of matrix metalloproteinases-2 and -9 . The results of the current study indicate that further evaluation of the mechanisms underlying the attenuation of MIA-induced OA by ETT are required, and may support the development of ETT as a potential therapeutic agent for the treatment of inflammatory diseases such as OA.

\section{Introduction}

Arthritis is a disorder involving the inflammation of one or more joints. The most common form of arthritis is osteoarthritis (OA), which is a degenerative joint disease characterized by progressive articular cartilage damage in multiple joints (1) and is associated with inflammation. Other forms include rheumatoid and psoriatic arthritis, which are classed as autoimmune diseases (2-6). OA is a major public health problem, and the occurrence of OA-associated disability is expected to increase globally in the aging population over the next decade, owing to increased life expectancy. Furthermore, OA is more prevalent with advancing age; however, studies on inflammation induced by various factors have demonstrated that it can occur in the young population as well $(7,8)$. OA is induced by several complex mechanisms, including progressive erosion of articular cartilage, destruction of joints and disruption of the cartilage network (9). It affects the articular cartilage as well as the whole joint, including the muscles and the subchondral bone $(10,11)$. Furthermore, it changes the subchondral bone formation, leading to sclerosis with underlying damaged cartilage and occasional joint inflammation, which alters the morphology of several cell types $(10,11)$. Currently, studies of complicated OA are performed in animal models, which monitor the disease progression and test drug candidates using imaging technology $(12,13)$. Previous studies demonstrated that monosodium iodoacetate (MIA), a metabolic inhibitor, induced OA following intra-articular injection. MIA has been reported to disrupt glycolysis, and the subsequent cell death and loss of chondrocytes results in morphological changes in the knee joint of animal models that are similar to those of human OA $(12,14,15)$.

Cyclooxygenase (COX)-2 belongs to the family of prostaglandin endoperoxide synthases and is involved in the conversion of arachidonic acid to prostaglandin $\mathrm{H}_{2}$, an important inflammatory mediator (16-18). COX-2 has been reported to be involved in a wide range of inflammatory 
diseases, and its inhibition has been actively studied. In particular, anti-inflammatory therapeutic agents have been developed, such as celecoxib, which is a specific inhibitor of COX-2. Drug development studies for the treatment of OA have also actively targeted COX-2 (19-21). Furthermore, nonsteroidal anti-inflammatory drugs (NSAIDs), which exhibit considerable efficacy in the treatment of OA, have been developed. However, the currently approved OA therapeutic agents frequently lead to gastrointestinal intolerance and irritation $(22,23)$. Therefore, further understanding of the molecular mechanisms of anti-inflammatory actions may facilitate the development of therapies for OA with minimal side-effects.

Tribulus terrestris L. is a member of the caltrop family (Zygophyllaceae) and it has been used as a traditional folk medicine for the treatment of inflammation, high blood pressure, edema, urinary infections and other ailments in China, India and other countries (24-27). Various phytochemical studies have demonstrated that $T$.terrestris contains flavonoids, steroidal saponins and lignanamides (28-32). In addition, studies have demonstrated that the extracts of $T$. terrestris have vasodilatory, anti-apoptotic and antioxidant properties, and may have effects on cardiovascular disease, hypertension, diabetes, cancer and fungal infections (33-38). Previous studies have also indicated that $T$. terrestris is effective in treating tracheitis, edema and inflammation, as well as protecting joints against inflammatory damage $(39,40)$. However, the effects and molecular mechanisms of $T$. terrestris on OA are currently unknown. Therefore, the present study evaluated the effects of the ethanolic extract of the fruits of T. terrestris (ETT) on an MIA-induced OA animal model, and aimed to elucidate the associated signaling pathways mediating its effect.

\section{Materials and methods}

Reagents. MIA was purchased from Sigma-Aldrich (Merck KGaA, Darmstadt, Germany). Rabbit anti-nitric oxide synthase (NOS) 2 (1:1,000; catalog no. 13120), rabbit anti-COX-1 (1:1,000; catalog no. 4841), rabbit anti-COX-2 (1:1,000; catalog no. 12282), rabbit anti-matrix metalloproteinase (MMP)-2 (1:1,000; catalog no. 4022), rabbit anti-MMP-9 (1:1,000; catalog no. 3852), rabbit anti-phospho-extracellular signal-regulated kinase (p-ERK; 1:1,000; T202/Y204; catalog no. 9101) and rabbit anti-ERK (1:1,000; catalog no. 9102) were purchased from Cell Signaling Technology, Inc. (Danvers, MA, USA), whereas the mouse anti- $\beta$-actin $(1: 1,000$; catalog no. sc-47778), and mouse (catalog no. sc-2005) and rabbit (catalog no. sc-2004) IgG-horseradish peroxidase-conjugated antibodies were purchased from Santa Cruz Biotechnology, Inc. (Dallas, TX, USA). Celebrex was purchased from Pfizer, Inc. (New York, NY, USA) and Joins was purchased from SK Chemicals (Seongnam, Korea).

Preparation of natural product extracts. The dried fruits of $T$. terrestris plant material were purchased from the Kyeongdong Oriental Herbal Store (Seoul, Korea) in March 2012, and identified by Professor Joa Sub Oh (College of pharmacy, Dankook University, Cheonan, Korea). A voucher specimen (G46) was deposited at the Bio-center herbarium of the Gyeonggi Institute of Science and Technology Promotion (Suwon, Korea). The air-dried and crushed fruits of $T$. terrestris $(10 \mathrm{~kg})$ were pulverized and extracted with $80 \%$ ethanol (EtOH; 3x181) at $24^{\circ} \mathrm{C}$ (twice a day for 2 days).

Animals and treatments. Female, 4-week-old LEW/SSNHSD rats (Envigo Ltd., Indianapolis, IN, USA) weighing 120-130 g $(n=50)$ at the start of the experiment, were used. They were kept in a sanitary ventilated animal room at a controlled temperature $\left(21 \pm 1^{\circ} \mathrm{C}\right)$ and a $12 \mathrm{~h}$ light-dark cycle, and were fed standard diets (Envigo Ltd.) with water available freely available. The procedures used in the current study complied with the guidelines of the Institutional Animal Care and Use Committee of Bio-center of the Gyeonggi Institute of Science and Technology Promotion. Following acclimatization to SPF system conditions for 1 week, rats were divided into six groups ( $n=7$ per group) as follows: Normal (injection of saline); vehicle (injection of $1.0 \mathrm{mg}$ MIA); MIA (1.0 mg) + ETT (200 mg/kg/day); MIA (1.0 mg) + ETT (400 mg/kg/day); MIA (1.0 mg) + Joins (200 mg/kg/day); and MIA (1.0 mg) + Celebrex (100 mg/kg/day). To induce arthritis with MIA, rats were anesthetized with $1 \%$ isoflurane in $\mathrm{O}_{2}$ at a flow rate of $1 \mathrm{l} / \mathrm{min}$ and a single percutaneous intra-articular injection of $1.0 \mathrm{mg}$ of MIA was administered, which was dissolved in physiological saline and administered in a $50 \mu \mathrm{l}$ volume using a 31 gauge 6-mm needle. Each experimental group was administered orally with either saline, 200 or $400 \mathrm{mg} / \mathrm{kg}$ ETT, $100 \mathrm{mg} / \mathrm{kg}$ Celebrex (Pfizer, Inc., NY, USA) or $200 \mathrm{mg} / \mathrm{kg}$ Joins (SK Chemicals) once daily for 4 weeks after the MIA injection. All experimental procedures in the protocol were approved by the Gyeonggi Institute of Science and Technology Promotion Institutional Animal Care and Ethical Use Committee (approval no. 2014-02-0005) and were in compliance with the Guide for the Care and Use of Laboratory Animals, Gyeonggi Institute of Science and Technology Promotion Institutional certificates, Association for Assessment and Accreditation of Laboratory Animal Care International (http://www.aaalac. org/resources/Guide_2011.pdf).

Micro-computed tomography (micro-CT). The in vivo micro-computed tomography (micro-CT) imaging was performed using a micro-CT (Inveon $^{\mathrm{TM}}$; Siemens Healthcare Ltd., Surrey, UK). Each rat was anesthetized with $1 \%$ isoflurane in $\mathrm{O}_{2}$ and placed on the scanner bed in a prone position. The total scanning time was 5 min during which the rat was anesthetized, and the scans were performed using the following settings: X-ray source voltage, $60 \mathrm{kVp}$; current, $400 \mu \mathrm{A}$; and a $1 \mathrm{~mm}$ thick aluminum filter was used to reduce beam-har dening artifact. The pixel size was $38.7 \mu \mathrm{m}$, exposure time was $400 \mathrm{~ms}$ and the rotation step was $1^{\circ}$ with a complete rotation over $201^{\circ}$. The $\mathrm{CT}$ images were reconstructed using COBRA reconstruction software version 6.3.39 (Exxim Computing Corporation; Pleasanton, CA, USA). The CT reconstruction protocol used a downsampling factor of 1 and was set to interpolate bilinearly using a Shepp-Logan filter. Siemens Inveon $^{\mathrm{TM}}$ Research Workplace software version 4.2; (Siemens Healthcare Ltd.) was used for visualization and analysis.

Bone morphometric analysis. The effect of OA treatment was investigated by bone morphometric analysis. Briefly, a cylinder was selected in the metaphysis of the tibia that contained the 
trabecular bone, and the bone volume was calculated. The bone volume fraction, which describes the ratio of bone volume to total volume (BV/TV) as well as the trabecular thickness (Tb.Th), the bone surface area over bone volume (BS/BV) and trabecular spacing (Tb.Sp) were determined for the trabecular bone. Siemens Inveon ${ }^{\mathrm{TM}}$ Research Workplace software version 4.2 (Siemens Healthcare Ltd.) was used for visualization and analysis.

Western blot analysis. Articular rat cartilage samples were collected 4 weeks after the MIA injection, pulverized and then lysed by incubation in $50 \mathrm{mM}$ Tris- $\mathrm{HCl}(\mathrm{pH} 7.4), 150 \mathrm{mM}$ sodium chloride $(\mathrm{NaCl}), 10 \%$ glycerol, $1 \%$ Triton X-100, $1 \mathrm{mM}$ EDTA, $100 \mu \mathrm{g} / \mathrm{ml}$ 4-(2-aminoethyl) benzenesulfonyl fluoride, $10 \mu \mathrm{g} / \mathrm{ml}$ aprotinin, $1 \mu \mathrm{g} / \mathrm{ml}$ pepstatin A, $0.5 \mu \mathrm{g} / \mathrm{ml}$ leupeptin, $80 \mathrm{mM} \beta$-glycerophosphate, $25 \mathrm{mM}$ sodium fluoride and $1 \mathrm{mM}$ sodium orthovanadate for $30 \mathrm{~min}$ at $4^{\circ} \mathrm{C}$. The tissue lysates were clarified by centrifugation at $12,500 \mathrm{x} \mathrm{g}$ for $20 \mathrm{~min}$ at $4^{\circ} \mathrm{C}$, and the supernatants were analyzed using western blot analysis. Total protein was quantified using a Bradford assay (Bio-Rad Laboratories, Hercules, CA, USA) with bovine serum albumin (BSA, Sigma-Aldrich) as the standard. A total of $40 \mu \mathrm{g}$ protein was loaded onto $10 \%$ gels and subjected to SDS-PAGE via the BioRad Mini Protean ${ }^{\circledR} 3$ system (Bio-Rad Laboratories), and transferred onto nitrocellulose membranes (Sigma-Aldrich). Membranes were blocked with 5\% BSA (Sigma-Aldrich) in PBS/0.025\% Tween 20 (PBST) for $1 \mathrm{~h}$ at room temperature. The primary antibodies used were specific for NOS2, COX-1, COX-2, MMP-2, MMP-9, p-ERK and ERK. These were diluted in 5\% BSA in PBST and incubated with the membranes overnight at $4{ }^{\circ} \mathrm{C}$. Secondary antibodies were applied at a 1:2,000 dilution in 5\% BSA in PBST and incubated for $1 \mathrm{~h}$ at room temperature. Membranes were subsequently processed for detection with the Supersignal ${ }^{\circledR}$ West Pico Chemiluminescent Substrate (Thermo Fisher Scientific, Inc., Waltham, MA, USA), using the Amersham ${ }^{\mathrm{TM}}$ Imager 600 and image analysis software version 0.4.4 (GE Healthcare Life Sciences, Chalfont, UK). Experiments were performed at least three times. Gels from one independent experiment are demonstrated in the present study.

$R N A$ purification and reverse transcription-polymerase chain reaction $(R T-P C R)$. Articular rat cartilage samples were pulverized and the total RNA was extracted using TRIzol ${ }^{\circledR}$ reagent (Invitrogen; Thermo Fisher Scientific, Inc.) according to the manufacturer's protocol. The integrity of the RNA was evaluated using agarose gel electrophoresis and ethidium bromide staining. Briefly, $1 \mu \mathrm{g}$ RNA was used as a template for each RT-PCR, using the SuperScript ${ }^{\circledR}$ III First-Strand Synthesis System and Platinum ${ }^{\circledR}$ PCR SuperMix which contains Taq DNA polymerase (Invitrogen; Thermo Fisher Scientific, Inc.), according to the manufacturer's protocol. Primers for the PCR were synthesized by Bioneer Corporation (Daejeon, Korea) and the sequences were as follows: COX-1, 5'-CTCACAGTG CGGTCCAAC-3' (forward) and 5'-CAGCACCTGGTACTT AAG-3' (reverse); COX-2, 5'-TGTATGCTACCATCTGGC TTCGG-3' (forward) and 5'-GTTTGGAACAGTCGCTCG TCATC-3' (reverse); NOS2, 5'-CATTGGAAGTGAAGCGTT TCG-3' (forward) and 5'-CAGCTGGGCTGTACAAACCTT-3' (reverse); tumor necrosis factor (TNF)- $\alpha, 5$ '-CCTCCCTCT
CATCAGTTCTA-3' (forward) and 5'-CAGAGAGGAGGT TGACTTTC-3' (reverse); interleukin (IL)-6, 5'-AGAGGAGAC TTCACAGAGGA-3' (forward) and 5'-CCAGTTTGGTAG CATCCATC-3' (reverse); MMP-2, 5'-ACATCTTTGCAGGAG ACAAG-3' (forward) and 5'-AAGCCACCCTCTTAAATC TG-3' (reverse); MMP-9, 5'-CGCTCATGTACCCGCTGTAT-3' (forward) and 5'-TGTCTGCCGGACTCAAAGAC-3' (reverse); and GAPDH, 5'-GTATGACTCCACTCACGGCAAA-3' (forward) and 5'-GGTCTCGCTCCTGGAAGATG-3' (reverse). RT-PCR amplification was performed using MyGene ${ }^{\mathrm{TM}}$ Series Peltier Thermal Cycler (LongGene ${ }^{\circledR}$ Scientific Instruments Co., Ltd., Hangzhou, China) and AccuPower ${ }^{\circledR}$ Pfu PCR PreMix (Bioneer Corporation). Cycling conditions were as follows: An initial predenaturation step at $95^{\circ} \mathrm{C}$ for $5 \mathrm{~min}$, followed by 25 cycles of denaturation at $95^{\circ} \mathrm{C}$ for $30 \mathrm{sec}$, annealing at 55 and $60^{\circ} \mathrm{C}$ for $40 \mathrm{sec}$, extension at $72^{\circ} \mathrm{C}$ for $60 \mathrm{sec}$, and a final extension step at $72^{\circ} \mathrm{C}$ for $10 \mathrm{~min}$. PCR products were electrophoresed on a $1 \%$ agarose gel and stained with ethidium bromide. Bands were captured using a ChemiDoc ${ }^{\mathrm{TM}}$ XRS system (Bio-Rad Laboratories) and quantified using Quantity One software version 4.6.3 (Bio-Rad Laboratories). Data was compared with the housekeeping gene GAPDH.

Zymogram analysis. The activities of the MMPs were measured using zymography (41). Briefly, proteins were extracted from pulverized cartilage tissue and separated using $8 \%$ SDS-PAGE with gels containing $1 \mathrm{mg} / \mathrm{ml}$ gelatin (Sigma-Aldrich). Gels were subsequently incubated in $2.5 \%$ Triton X-100 for $1 \mathrm{~h}$ at room temperature to remove the SDS and renature the MMPs, and were incubated in developing buffer containing $50 \mathrm{mM}$ Tris- $\mathrm{HCl}(\mathrm{pH} \mathrm{7.5}), 10 \mathrm{mM}$ calcium chloride, and $150 \mathrm{mM} \mathrm{NaCl}$ for $16 \mathrm{~h}$ at $37^{\circ} \mathrm{C}$. The gels were stained with $0.5 \%$ Coomassie Brilliant Blue R-250 in methanol:acetic acid (30:10) for $3 \mathrm{~h}$ at room temperature, followed by destaining with methanol:acetic acid (30:10). The gelatinolytic activities were detected as unstained bands against the background of the Coomassie Blue-stained gel.

Histology. For histological analysis, rat knee joints were removed, fixed in 10\% neutral- buffered formalin and subsequently decalcified with $10 \%$ formic acid for $48 \mathrm{~h}$ at room temperature and embedded in paraffin. Subsequently, $5 \mu \mathrm{m}$ thick frontal sections of the medial aspect of the knee joints were cut. Hematoxylin and eosin (H\&E) staining was performed to investigate inflammatory infiltrates in the knee joints and surrounding tissues, and Masson's trichrome staining was performed to determine the degeneration of cartilage. Staining was performed at room temperature and tissues were observed under a light microscope.

Statistical analysis. Statistical analysis was performed by a Student's t-test using Microsoft Excel 2007 software (Microsoft Corporation, Redmond, WA, USA). Results are presented as the mean \pm standard deviation. $\mathrm{P}<0.05$ was considered to indicate a statistically significant difference.

\section{Results}

Cartilage and subchondral bone changes. To investigate the effects of ETT in OA, an MIA rat model of OA was established 
A
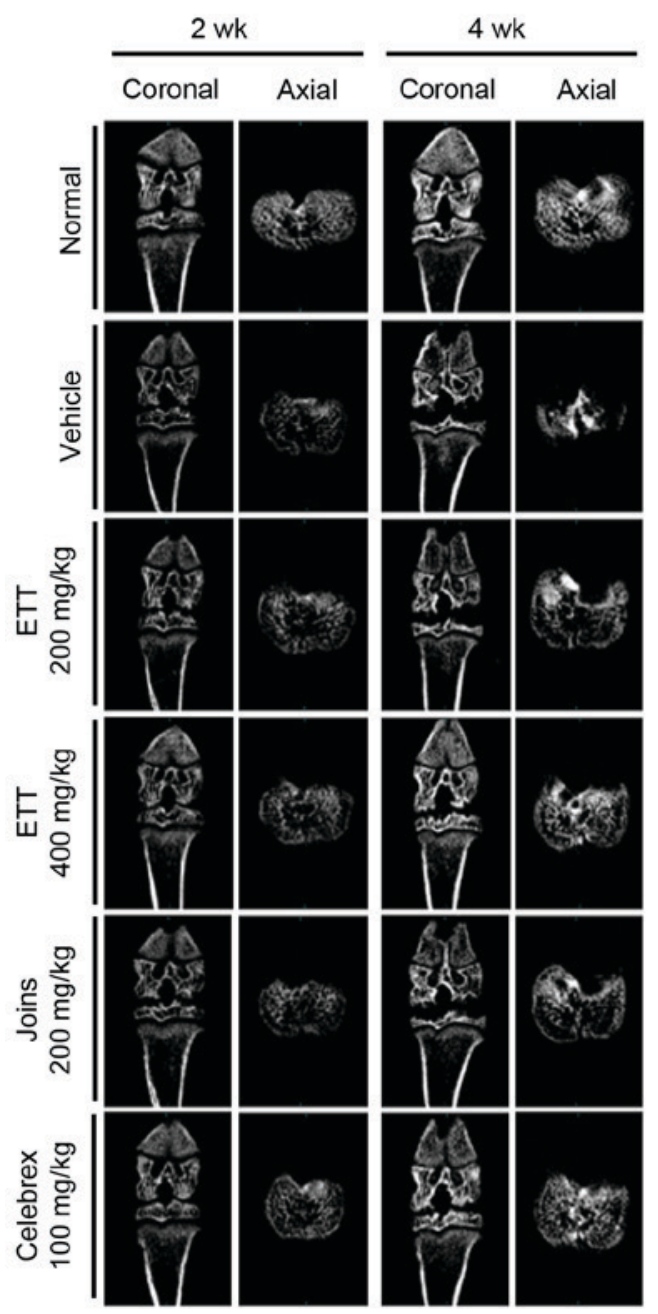

C
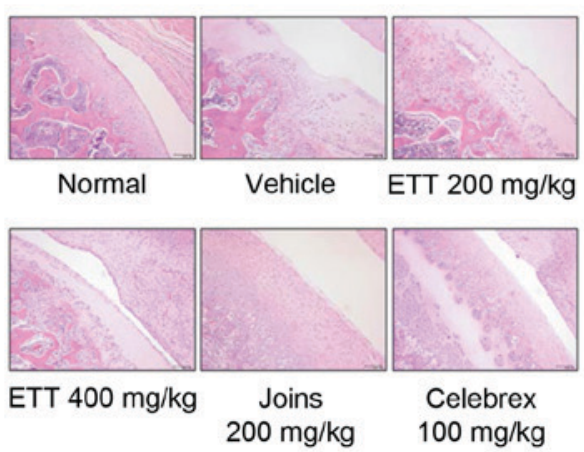

B
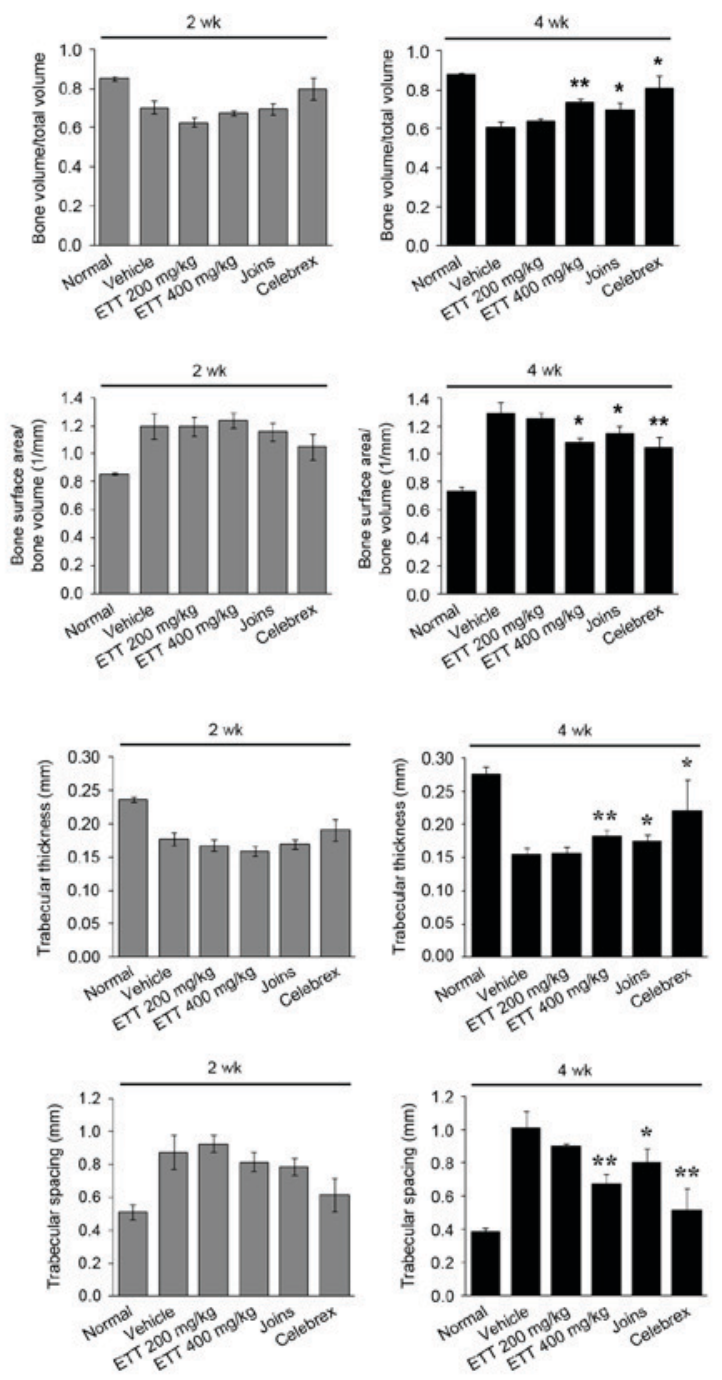

D
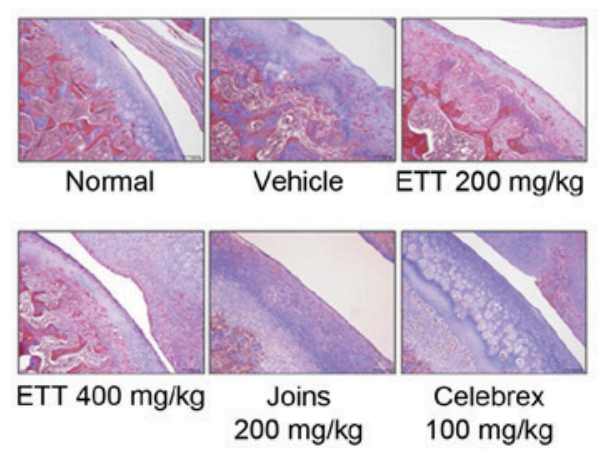

Figure 1. Morphological and histological results in a MIA-induced rat OA model. (A) Micro-CT images of MIA-injected joints demonstrate severe damage with rough edges around the tibia and femur, indicative of bone lysis and swelling in the vehicle group. Damage was visibly reduced by treatment with ETT, Joins and Celebrex (400, 200 and $100 \mathrm{mg} / \mathrm{kg}$, respectively). (B) Morphometric parameters of tibial trabecular bone determined using micro-CT. After 2 weeks, no significant differences in morphometric parameters were observed in the trabecular bone region between vehicle and treated groups. At 4 weeks post-injection, BV/TV and Tb.Th were significantly increased, whereas BS/BV and Tb.Sp were significantly decreased by treatment with ETT, Joins and Celebrex (400, 200 and $100 \mathrm{mg} / \mathrm{kg}$, respectively), compared with the vehicle group. Histopathological analysis of an OA lesion in the knee joint of rats 4 weeks after intra-articular injection of MIA presenting (C) hematoxylin and eosin staining and (D) Masson's trichrome staining of the cartilage of knee joints of rats 4 weeks after MIA injection. Magnification, $\mathrm{x} 25 .{ }^{*} \mathrm{P}<0.05$ and ${ }^{* *} \mathrm{P}<0.01$ vs. vehicle group. MIA, monosodium iodoacetate; OA, osteoarthritis; micro-CT, micro-computed tomography; ETT, ethanolic extract of Tribulus terrestris; BV/TV, bone volume fraction; Tb.Th, trabecular thickness; BS/BV, bone surface area/bone volume; Tb.Sp, trabecular spacing; wk, weeks.

as described in the materials and methods section. The rats were subjected to the first micro-CT scan at 2 and 4 weeks after the MIA injection, and the cartilage damage and subchondral bone erosions in the knee joints were visualized (Fig. 1A).
All rats exhibited pathological bone changes in the MIA-injected knee after 4 weeks, whereas the normal knees had no OA-like changes in the bone throughout the study. The morphological changes were more evident and the cartilage 
A

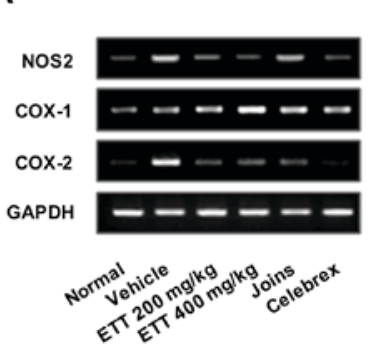

B

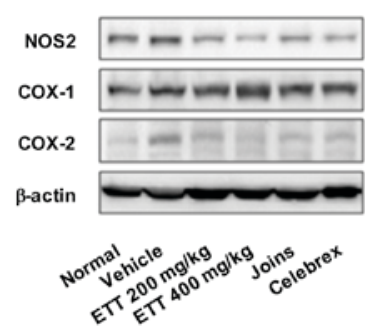

Nos2

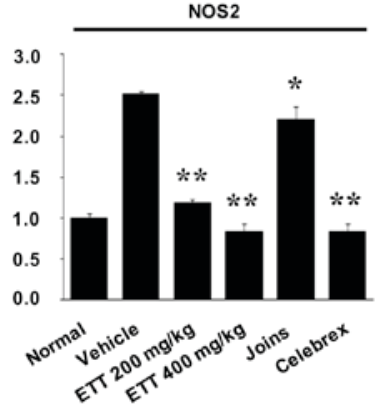

Nos2

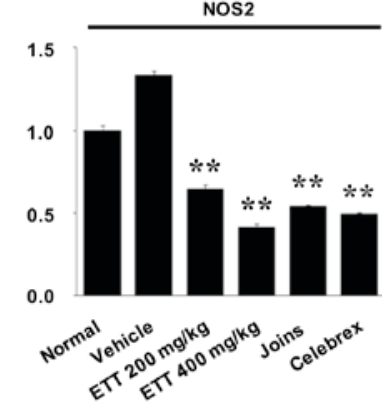

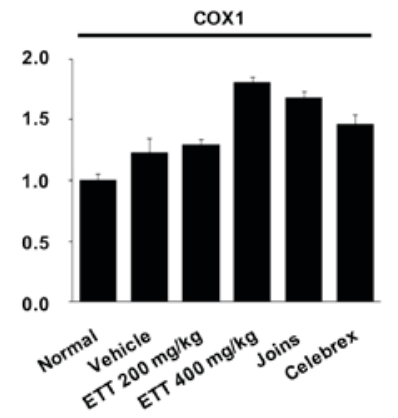
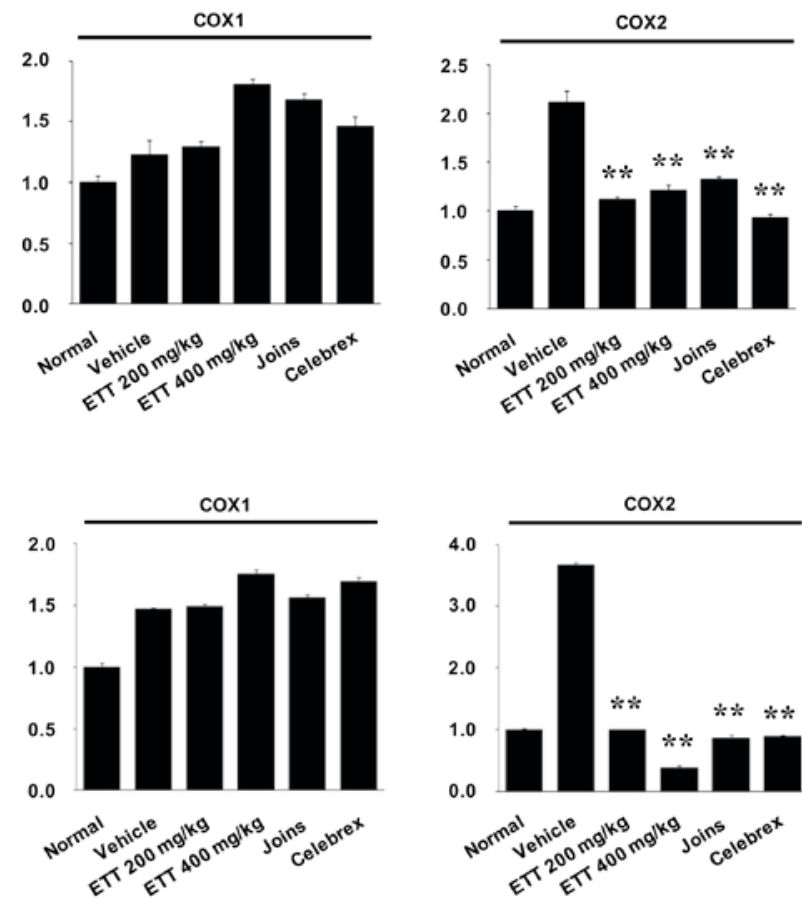

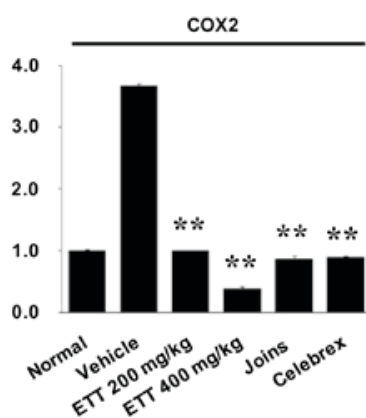

Figure 2. ETT suppressed MIA-induced inflammatory enzyme expression. (A) COX-2 and NOS2 mRNA and quantification. (B) Protein expression in the cartilage of rat knee joints 4 weeks after MIA injection. Densitometric analysis of western blot results for NOS2, COX-1 and COX-2 is demonstrated. Results represent at least three independent experiments. ${ }^{*} \mathrm{P}<0.05$ and ${ }^{* *} \mathrm{P}<0.01$ vs. vehicle group. ETT, ethanolic extract of Tribulus terrestris; MIA, monosodium iodoacetate; COX, cyclooxygenase; NOS, nitric oxide synthase.

exhibited rougher edges in the vehicle group (MIA injection only) compared with the treated groups. Morphological changes in the vehicle group were visibly reduced by treatment with ETT (400 mg/kg) and Celebrex (100 mg/kg). The basic method of quantitatively describing bone architecture involves the calculation of morphometric parameters, which can be computed using software available from several micro-CT manufacturers. The BV/TV is the ratio of the segmented BV to the TV of the region of interest, and the specific BS (BS/BV) is the ratio of the segmented BS to the segmented BV. The Tb.Th and Tb.Sp values denote the thickness of the trabeculae and the distance in between the trabeculae, which were assessed using direct 3D methods $(15,42)$.

Bone structure analysis 4 weeks after the MIA injection demonstrated marked changes in the architecture, which were indicative of osteoarthritic joint destruction. At 4 weeks, there was a significant increase in the BV/TV and Tb.Th of the ETT $400 \mathrm{mg} / \mathrm{kg}$-, Joins- and Celebrex-treated groups compared with the vehicle-treated group $(\mathrm{P}<0.05$; Fig. $1 \mathrm{~B})$. In addition, the Tb.Sp and BS/BV were significantly lower in the ETT $400 \mathrm{mg} / \mathrm{kg}$-, Joins- and Celebrex-treated groups compared with the vehicle-treated group $(\mathrm{P}<0.05$; Fig. $1 \mathrm{~B})$. Therefore, MIA induction of OA-like effects was reduced by ETT treatment. Furthermore, at 2 weeks, no statistically significant differences were observed in the structural parameters of all the groups. At 4 weeks after the MIA treatment, H\&E staining revealed cartilage damage accompanied by structural degradation and loss of chondrocytes (Fig. 1C). However, the cartilage damage was reduced following treatment with ETT $(400 \mathrm{mg} / \mathrm{kg}$ ) compared with the vehicle-treated group. Masson's trichrome staining also revealed proteoglycan and collagen degradation of the cartilage tissue of MIA-injected rats in the vehicle group (Fig. 1D). In addition, the degradation of proteoglycan and collagen was reduced by treatment with ETT $(400 \mathrm{mg} / \mathrm{kg}$ ) compared with the vehicle-treated group. The results indicate that ETT reduced MIA-induced OA, and the effects of ETT at $400 \mathrm{mg} / \mathrm{kg}$ were similar to those of Joins and Celebrex.

ETT suppresses MIA-induced OA by modulating NOS2 and $C O X-2$ expression. The regulatory effect of ETT on MIA-induced OA was examined by analyzing the changes in NOS2 and COX-2 expression, which are important mediators of inflammation and the development of OA requires their induction $(43,44)$. To examine the inhibitory effects of ETT on mRNA and protein expression of NOS2 and COX-2, RT-PCR and western blot analysis was performed and results are presented in Fig. 2. ETT markedly suppressed the mRNA (Fig. 2A) and protein (Fig. 2B) expression of NOS2 and COX-2 in the MIA-induced OA tissue compared with the vehicle-treated group. In addition, treatment with Joins and Celebrex suppressed the expression of NOS2 and COX-2 in response to MIA stimulation, indicating that ETT may contain pharmacologically effective components and possess mechanisms of action that are similar to those of the standard therapeutic agents, in regulating MIA-induced OA. The results indicate that ETT downregulated the expression of NOS2 and COX-2 proteins compared with the vehicle-treated group, and thereby attenuated MIA-induced OA.

Anti-OA activities of ETT are mediated by inhibiting inflammatory cytokines and mitogenic signaling pathways. To further investigate the molecular mechanisms by which ETT inhibits OA, the changes in the mRNA expression and 
A

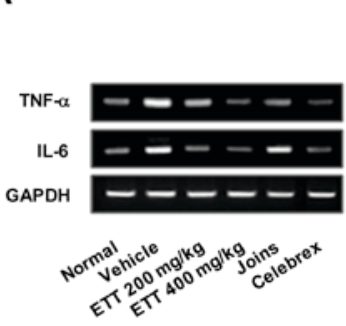

B

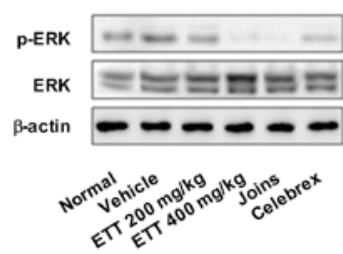

TNF- $\alpha$
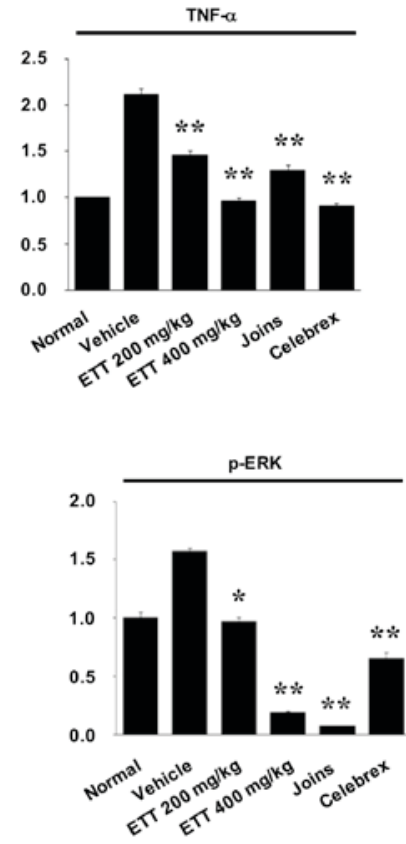

Figure 3. ETT suppressed pro-inflammatory cytokine expression in MIA-induced osteoarthritis. (A) TNF- $\alpha$ and IL-6 mRNA expression and quantification (B) Phosphorylated ERK protein levels in the cartilage of rat knee joints 4 weeks after MIA injection, as demonstrated by western blotting and densitometric analysis. Results represent at least three independent experiments. " $\mathrm{P}<0.05$ and ${ }^{* *} \mathrm{P}<0.01$ vs. vehicle group. ETT, ethanolic extract of Tribulus terrestris; MIA, monosodium iodoacetate; TNF- $\alpha$, tumor necrosis factor- $\alpha$; IL, interleukin; ERK, extracellular signal-regulated kinase; p-ERK, phosphorylated ERK.

A

B

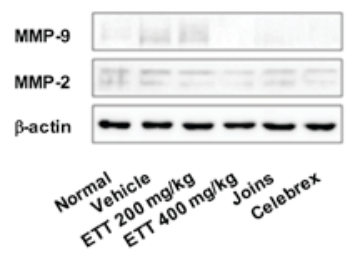

C

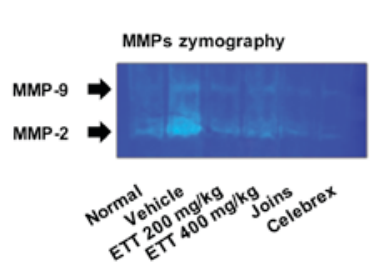

MMP-9

MMP-2

GAPDH

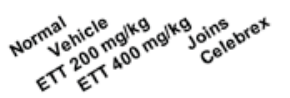

(1)
MMP-9

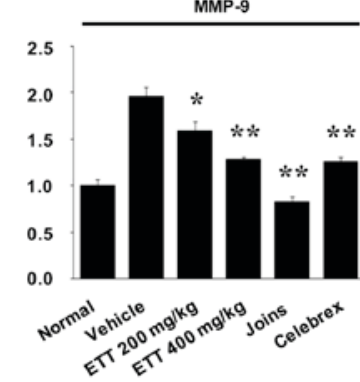

MMP9

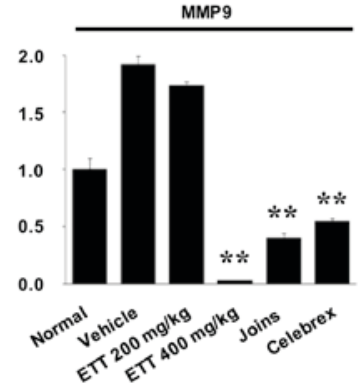

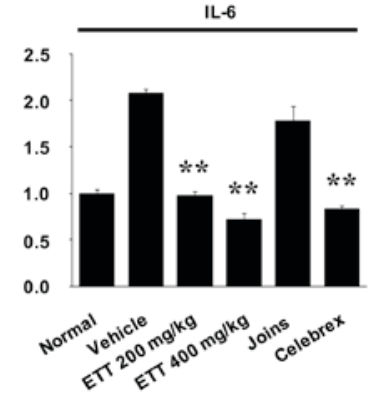
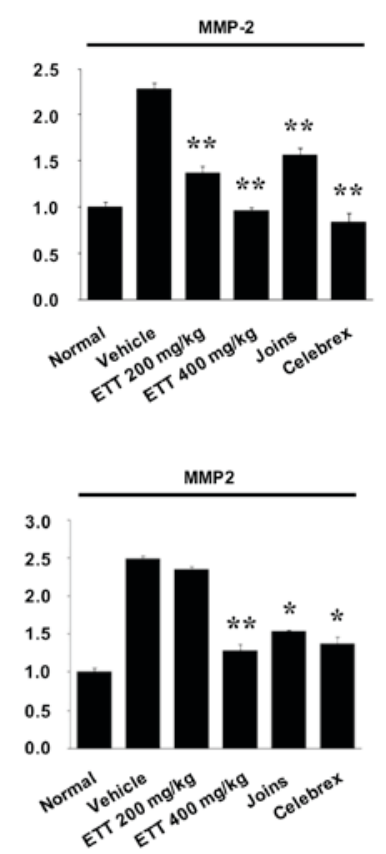

Figure 4. ETT reduced MIA-induced MMP-9 and MMP-2 expression. (A) MMP-9 and MMP-2 mRNA and quantification. (B) Protein expression in the cartilage of rat knee joints 4 weeks after MIA injection, as demonstrated by western blotting and densitometric analysis. (C) Gelatin zymogram analysis using tissue lysates. Zymogram gel loading was normalized to total protein concentration. Results represent at least three independent experiments. ${ }^{*}<0.05$ and ${ }^{* * *} \mathrm{P}<0.01$ vs. vehicle group. ETT, ethanolic extract of Tribulus terrestris; MIA, monosodium iodoacetate; MMP, metalloproteinase. 
the phosphorylation of inflammatory cytokines, including TNF- $\alpha$, IL- 6 and ERK, which play pivotal roles in inflammation $(42,45,46)$ were examined. As demonstrated in Fig. 3, MIA stimulation markedly increased the mRNA expression of TNF- $\alpha$ and IL-6, and the phosphorylation of ERK compared with the untreated group. By contrast, ETT treatment reduced the expression of TNF- $\alpha$ and IL-6, and the phosphorylation of ERK compared with the vehicle-treated group. Although certain differences were observed compared with Joins and Celebrex, the inhibitory effect of ETT was similar to these agents. Combined, the results demonstrate that the suppression of OA by ETT may be mediated by the inhibition of the expression of inflammatory cytokines, including TNF- $\alpha$ and IL-6, as well as inactivation of ERK signaling pathways.

ETT suppresses MMP-2 and MMP-9 expression in MIA-induced OA. The expression and activation of MMPs has been reported to promote inflammatory responses, including rheumatoid arthritis and OA. In particular, MMP-2 and MMP-9 have been previously reported to be associated with OA (47). To examine the molecular mechanism underlying the regulatory effects of ETT in OA, the changes in the mRNA and protein expression of MMP-2 and MMP-9 in MIA-induced OA was examined, and it was observed that ETT treatment dose-dependently reduced their expression compared with the vehicle-treated group (Fig. 4A and B). In addition, Fig. 4C demonstrates that ETT treatment reduced MIA-induced activation of MMP-2 and MMP-9 in MIA-induced OA tissue. This inhibitory effect of ETT was similar to that observed following treatment with Joins and Celebrex. Collectively, the results indicate that the inhibitory effects of ETT in MIA-induced OA may be mediated by the suppression of MMP-2 and MMP-9 expression.

\section{Discussion}

T. terrestris is used as a traditional medicine and has been demonstrated to be effective in the treatment of various conditions, including diabetes, cardiovascular and fungal disease. These effects are supported by a previous study that revealed antioxidant, antiedema and anti-inflammatory activities of $T$. terrestris $(37,39,40)$. However, to the best of our knowledge, the present study is the first to report the effects and molecular mechanisms of action of T. terrestris against OA.

The current study investigated the protective effects of ETT on cartilage and its molecular mechanisms of action in an MIA-induced OA rat model. Micro-CT has revolutionized the application of animal models in arthritis research and has become the standard for the evaluation of bone morphology and bone structure in these models. Furthermore, the advance in 3D imaging techniques, such as micro-CT, has provided an accurate, non-invasive tool for measuring microstructure (48). Therefore, the present study performed micro-CT to confirm the cartilage protective effect of ETT treatment. A previous study reported a decrease in the BV/TV and Tb.Th, with an increase in BS/BV and Tb.Sp in OA following treatment (49), results which were similar to the results obtained in the present study. Specifically, the present study demonstrated that the BV/TV and Tb.Th were significantly increased, while the $\mathrm{BS} / \mathrm{BV}$ and Tb.Sp were significantly decreased in the ETT
$400 \mathrm{mg} / \mathrm{kg}$-treated group compared with the vehicle-treated group. Therefore, ETT treatment (400 mg/kg) enhanced the maintenance of the BV/TV and Tb.Th, as well as the recovery of BS/BV and Tb.Sp compared with Joins treatment (Fig. 1).

In our previous study, we demonstrated for the first time that two new furostanol glycosides, termed terrestinones A1/A2 (1a/1b), isolated from ETT (50), and N-trans- $\rho$-caffeoyl tyramine isolated from ETT, inhibited macrophage-mediated inflammatory responses (51). Furthermore, our previous in vitro data demonstrated that the anti-inflammatory effects of $\mathrm{N}$-trans- $\rho$-caffeoyl tyramine were mediated by the regulation of pro-inflammatory cytokines, including TNF- $\alpha$, IL-6, IL-10, NO, COX-2 and c-Jun N-terminal kinase activity in lipopolysaccharide-stimulated RAW 264.7 cells (51). These in vitro results support the therapeutic usefulness of ETT for development as a treatment for OA. Similar to the previous results, the present study confirmed that ETT inhibited MIA-induced NOS2, COX-2, TNF- $\alpha$ and IL- 6 mRNA and protein expression, as well as the activation of ERK in our in vivo OA model (Figs. 2 and 3 ).

MMPs belong to a family of zinc-dependent endopeptidases that regulate the proteolytic degradation of the extracellular matrix and have a pivotal role in various processes, including cancer progression, tumor angiogenesis and the release of growth factors $(52,53)$. Previous studies have reported that MMPs are associated with OA and, in particular, MMP2 and MMP9 have been reported to be closely involved. The levels of MMP-2 have been reported to increase in OA cartilage and bone, which are the gene products of human adult articular chondrocytes (54). MMP-9 was previously demonstrated to be highly expressed in severe OA, which was associated with high blood vessel infiltration compared with early OA (55). The present study demonstrated that ETT treatment dose-dependently reduced MIA-induced mRNA and protein expression, and activation of MMP-2 and MMP-9 (Fig. 4). In addition, the improvements in the BV/TV ratio and Tb.Th, as well as the decrease in the BS/BV and Tb.Sp, caused by Celebrex were observed to accompany a decrease in tissue MMPs, which is a biochemical marker of bone remodeling (56-58). Furthermore, the results of the present study indicate that COX-2 inhibition may reduce osteoclast activation caused by MMP-2 and MMP-9. In addition, COX-2 is involved in the inhibition of bone destruction, which is associated with the changes in trabecular microstructure associated with the progression of OA.

The present study demonstrates for the first time that ETT attenuated MIA-induced OA. Furthermore, the anti-OA activities of ETT were demonstrated to be mediated by the inhibition of MIA-induced expression of NOS2, COX-2, TNF- $\alpha$ and IL-6. In addition, ETT markedly suppressed the phosphorylation of ERK, and decreased MIA-induced expression and activation of MMP-2 and MMP-9. Notably, the effects of ETT on the MIA-induced OA were similar to those of the control drugs, Joins and Celebrex. Joins is a complex natural product consisting of Prunella vulgaris, Trichosanthes kirilowii root extract and Clematis florida, while Celebrex is a prescription drug, which inhibits COX-2. Current treatment options for OA are limited, and include symptom management with simple analgesics, NSAIDs, corticosteroids and opiates. Non-pharmacological therapies range from physical exercise and weight loss to joint lavage and eventually, surgical 
intervention may be employed (59). Although NSAIDs are reasonably safe drugs, they all possess potential side-effects, which include heart attack, stroke and stomach bleeding (60). Treatment of OA has increasingly involved the use of COX-2 inhibitors more than NSAIDs (61). Therefore, ETT may be a useful candidate for the development of the first single natural-based medicine for the treatment of OA.

In conclusion, the present study investigated the effects of the ETT on MIA-induced OA and demonstrated that it reduced MIA-induced cartilage damage and the erosion of several bone surfaces using micro-CT. In addition, the effect of the ETT was confirmed to be similar to that of the COX-2 inhibitor, Celebrex. Furthermore, the anti-OA activity of ETT is potentially mediated by the downregulation of NO, NOS2, COX-2, TNF- $\alpha$ and IL-6. Furthermore, ETT-mediated attenuation of OA may also be dependent on the expression of MMP-2 and MMP-9. The results of the present study indicate that ETT has useful effects and has demonstrated potential for development as a novel drug for the treatment of the inflammation associated with $\mathrm{OA}$ and, therefore, warrants further investigation.

\section{Acknowledgements}

This research was supported by a grant from the High Value-Added Food Technology Development Program (grant no. 111130-03-1-HD110; Ministry for Agriculture, Food and Rural Affairs, Sejong, Korea).

\section{References}

1. Sniekers YH, Intema F, Lafeber FP, van Osch GJ, van Leeuwen JP, Weinans $\mathrm{H}$ and Mastbergen SC: A role for subchondral bone changes in the process of osteoarthritis; a micro-CT study of two canine models. BMC Musculoskelet Disord 9: 20, 2008.

2. Dancevic CM and McCulloch DR: Current and emerging therapeutic strategies for preventing inflammation and aggrecanase-mediated cartilage destruction in arthritis. Arthritis Res Ther 16: 429, 2014.

3. Mc Ardle A, Flatley B, Pennington SR and FitzGerald O: Early biomarkers of joint damage in rheumatoid and psoriatic arthritis. Arthritis Res Ther 17: 141, 2015.

4. Mellado M, Martínez-Muñoz L, Cascio G, Lucas P, Pablos JL and Rodríguez-Frade JM: T cell migration in rheumatoid arthritis. Front Immunol 6: 384, 2015.

5. Parsonage G, Filer A, Bik M, Hardie D, Lax S, Howlett K, Church LD, Raza K, Wong SH, Trebilcock E, et al: Prolonged, granulocyte-macrophage colony-stimulating factor-dependent, neutrophil survival following rheumatoid synovial fibroblast activation by IL-17 and TNFalpha. Arthritis Res Ther 10: R47, 2008.

6. van Osch GJ: Osteoarthritis year in review 2014: Highlighting innovations in basic research and clinical applications in regenerative medicine. Osteoarthritis Cartilage 22: 2013-2016, 2014.

7. Goldring MB and Otero M: Inflammation in osteoarthritis. Curr Opin Rheumatol 23: 471-478, 2011.

8. Simon AK, Seipelt E and Sieper J: Divergent T-cell cytokine patterns in inflammatory arthritis. Proc Natl Acad Sci USA 91: 8562-8566, 1994.

9. Choi JH, Choi JH, Kim DY, Yoon JH, Youn HY, Yi JB, Rhee HI, Ryu KH, Jung K, Han CK, et al: Effects of SKI 306X, a new herbal agent, on proteoglycan degradation in cartilage explant culture and collagenase-induced rabbit osteoarthritis model. Osteoarthritis Cartilage 10: 471-478, 2002.

10. Hinton R, Moody RL, Davis AW and Thomas SF: Osteoarthritis: Diagnosis and therapeutic considerations. Am Fam Physician 65: 841-848, 2002.

11. Little CB and Zaki S: What constitutes an 'animal model of osteoarthritis'-the need for consensus? Osteoarthritis Cartilage 20: 261-267, 2012.
12. Beyreuther B, Callizot N and Stöhr T: Antinociceptive efficacy of lacosamide in the monosodium iodoacetate rat model for osteoarthritis pain. Arthritis Res Ther 9: R14, 2007.

13. Piscaer TM, van Osch GJ, Verhaar JA and Weinans H: Imaging of experimental osteoarthritis in small animal models. Biorheology 45: 355-364, 2008.

14. Guzman RE, Evans MG, Bove S, Morenko B and Kilgore K: Mono-iodoacetate-induced histologic changes in subchondral bone and articular cartilage of rat femorotibial joints: An animal model of osteoarthritis. Toxicol Pathol 31: 619-624, 2003.

15. Mohan G, Perilli E, Kuliwaba JS, Humphries JM, Parkinson IH and Fazzalari NL: Application of in vivo micro-computed tomography in the temporal characterisation of subchondral bone architecture in a rat model of low-dose monosodium iodoacetate-induced osteoarthritis. Arthritis Res Ther 13: R210, 2011.

16. Hla T and Neilson K: Human cyclooxygenase-2 cDNA. Proc Natl Acad Sci USA 89: 7384-7388, 1992.

17. Vane JR, Mitchell JA, Appleton I, Tomlinson A, Bishop-Bailey D, Croxtall $\mathrm{J}$ and Willoughby DA: Inducible isoforms of cyclooxygenase and nitric-oxide synthase in inflammation. Proc Natl Acad Sci USA 91: 2046-2050, 1994.

18. Tsatsanis C, Androulidaki A, Venihaki M and Margioris AN: Signalling networks regulating cyclooxygenase-2. Int J Biochem Cell Biol 38: 1654-1661, 2006.

19. Gierse JK, McDonald JJ, Hauser SD, Rangwala SH, Koboldt CM and Seibert K: A single amino acid difference between cyclooxygenase-1 (COX-1) and -2 (COX-2) reverses the selectivity of COX-2 specific inhibitors. J Biol Chem 271: 15810-15814, 1996.

20. Hawkey CJ: COX-2 inhibitors. Lancet 353: 307-314, 1999.

21. Luong C, Miller A,Barnett J, Chow J, Ramesha C and Browner MF: Flexibility of the NSAID binding site in the structure of human cyclooxygenase-2. Nat Struct Biol 3: 927-933, 1996.

22. Kim JK, Park SW, Kang JW, Kim YJ, Lee SY, Shin J, Lee S and Lee SM: Effect of GCSB-5, a herbal formulation, on monosodium iodoacetate-induced osteoarthritis in rats. Evid Based Complement Alternat Med 2012: 730907, 2012.

23. Silverstein FE, Faich G, Goldstein JL, Simon LS, Pincus T, Whelton A, Makuch R, Eisen G, Agrawal NM, Stenson WF, et al: Gastrointestinal toxicity with celecoxib vs nonsteroidal anti-inflammatory drugs for osteoarthritis and rheumatoid arthritis: The CLASS study: A randomized controlled trial. Celecoxib Long-term Arthritis Safety Study. JAMA 284: 1247-1255, 2000 .

24. Aldein S: Medicinal, Herbs. First edition Baghdad: Dar Al-Shoun Al-Thaqafia Al-Aama for Publishing, pp70, 1986 (In Arabic).

25. CHEMEXCIL: Tribulus terrestris Linn. (N.O.-Zygophyllaceae) In: Selected medicinal plants of India (A monograph of identity, safety and clinical usage). Compiled by Bharatiya Vidya Bhavan's Swamy Prakashananda Ayurveda Research Centre for CHEMEXCIL. Tata Press, Bombay (Chapter 10), pp323-326, 1996.

26. Chopra RN, Chopra IC, Handa KL and Kapoor LD: Chopra's indigenous drugs of India. Second ed. U.N. Dhur \& Sons Private Ltd., Calcutta, 430-431, 1959.

27. Majeed SH and Mahmood MJ: Herbs and medicinal plants in Iraq between traditional medicine and scientific research. First edition Baghdad: Dar Al-Thaowra for Publishing, pp40, 1988 (In Arabic).

28. Mahato SB, Sahu NP and Pal BC: Screening of Tribulus terrestris plants for diosgenin. J Ind Chem (India) 50: 49-50, 1978.

29. Saleh NAM, Ahmed AA and Abdalla MF: Flavonoid glycosides of Tribulus pentandrus and T. terrestris. Phytochemistry 21: 1995-2000, 1982.

30. Tomowa MP and Gjulemetowa R: Steroidal saponins and steroidal sapogenins VI. Furostanol glycoside from Tribulus terrestris. Planta Med 34: 188-191, 1978.

31. Yan W, Ohtani K, Kasai R and Yamasaki K: Steroidal saponin from fruits of Tribulus terrestris. Phytochemistry 42: 1417-1422, 1996.

32. Zafar R and Lalwani M: Tribulus terrestris Linn. A review of current knowledge. Indian Drugs 27: pp148-153, 1989.

33. Capoor LD: Handbook of Ayurvedic Medicinal Plants. Boca Raton, New York, Washington: CRC Press, pp325-332, 1990.

34. Gauthaman K and Ganesan AP: The hormonal effects of Tribulus terrestris and its role in the management of male erectile dysfunction-an evaluation using primates, rabbit and rat. Phytomedicine 15: 44-54, 2008.

35. Jameel M, Ansari JA, Ali A, Ahamad J, Ali M and Tamboli E: Pharmacological scientific evidence for the promise of Tribulus terrestris. Int Res J Pharm 3: 403-406, 2012. 
36. Li H, Miao SB, Dong LH, Shu YN, Shao DC, Chen BC, Han M and Zhang Y: Clinicopathological correlation of Krüppel-like factor 5 and matrix metalloproteinase- 9 expression and cartilage degeneration in human osteoarthritis. Pathol Res Pract 208: 9-14, 2012.

37. Walczak-Jedrzejowska R, Wolski JK and Slowikowska-Hilczer J: The role of oxidative stress and antioxidants in male fertility. Cent European J Urol 66: 60-67, 2013.

38. Zheleva-Dimitrova D, Obreshkova D and Nedialkov $P$ Antioxidant activity of Tribulus terrestris-A natural product in infertility therapy. Int J Pharm Pharm Sci 4: 508-511, 2012.

39. Baburao B, Rajyalakshmi G, Venkatesham A, Kiran G, Shyamsunder A and Gangarao B: Anti-inflammatory and antimicrobial activities of methanolic extract of Tribulus terrestris Linn plant. Int J Chem Sci 7: 1867-1872, 2009.

40. Mishra NK, Biswal GS, Chowdary KA and Mishra G: Anti-arthritic activity of Tribulus terrestris studied in Freund's adjuvant induced arthritic rats. J Pharm Educ Res 4: 41-46, 2013.

41. Cho YR, Choi SW and Se DW: The in vitro antitumor activity of Siegesbeckia glabrescens against ovarian cancer through suppression of receptor tyrosine kinase expression and the signaling pathways. Oncol Rep 30: 221-226, 2013.

42. Papayianni A: Cytokines, growth factors, and other inflammatory mediators in glomerulonephritis. Ren Fail 18: 725-740, 1996.

43. Abramson SB: The role of COX-2 produced by cartilage in arthritis. Osteoarthritis Cartilage 7: 380-381, 1999.

44. Vane JR, Bakhle YS and Botting RM: Cyclooxygenases 1 and 2. Annu Rev Pharmacol Toxicol 38: 97-120, 1998.

45. Barton BE: IL-6: Insights into novel biological activities. Clin Immunol Immunopathol 85: 16-20, 1997.

46. Su B and Karin M: Mitogen-activated protein kinase cascades and regulation of gene expression. Curr Opin Immunol 8: 402-411, 1996

47. Galasso O, Familiari F, De Gori M and Gasparini G: Recent findings on the role of gelatinases (matrix metalloproteinase- 2 and -9) in osteoarthritis. Adv Orthop 2012: 834208, 2012.

48. Bouxsein ML, Boyd SK, Christiansen BA, Guldberg RE, Jepsen KJ and Müller R: Guidelines for assessment of bone microstructure in rodents using micro-computed tomography. J Bone Miner Res 25: 1468-1486, 2010

49. Kamibayashi L, Wyss UP, Cooke TD and Zee B: Trabecular microstructure in the medial condyle of the proximal tibia of patients with knee osteoarthritis. Bone 17: 27-35, 1995.

50. Hong SS, Choi YH, Jeong W, Kwon JG, Kim JK, Seo C, Ahn EK, Lee HH, Ko HJ, Seo DW and Oh JS: Two new furostanol glycosides from the fruits of Tribulus terrestris. Tetrahedron Lett 54: 3967-3970, 2013.
51. Ko HJ, Ahn EK and Oh JS: N-trans- $\rho$-caffeoyl tyramine isolated from Tribulus terrestris exerts anti-inflammatory effects in lipopolysaccharide-stimulated RAW 264.7 cells. Int J Mol Med 36: 1042-1048, 2015.

52. Kessenbrock K, Plaks V and Werb Z: Matrix metalloproteinases: Regulators of the tumor microenvironment. Cell 141: 52-67, 2010

53. Stetler-Stevenson WG: Matrix metalloproteinases in angiogenesis: A moving target for therapeutic intervention. J Clin Invest 103: 1237-1241, 1999.

54. Duerr S, Stremme S, Soeder S, Bau B and Aigner T: MMP-2/gelatinase A is a gene product of human adult articular chondrocytes and is increased in osteoarthritic cartilage. Clin Exp Rheumatol 22: 603-608, 2004.

55. Li SC: Chinese Medicinal Herbs. San Francisco, Georgetown Press, pp441, 1983

56. Hill PA, Murphy G, Docherty AJ, Hembry RM, Millican TA, Reynolds JJ and Meikle MC: The effects of selective inhibitors of matrix metalloproteinases (MMPs) on bone resorption and the identification of MMPs and TIMP-1 in isolated osteoclasts. J Cell Sci 107: 3055-3064, 1994.

57. Raggatt LJ and Partridge NC: Cellular and molecular mechanisms of bone remodeling. J Biol Chem 285: 25103-25108, 2010.

58. Zweers MC, de Boer TN, van Roon J, Bijlsma JW, Lafeber FP and Mastbergen SC: Celecoxib: Considerations regarding its potential disease-modifying properties in osteoarthritis. Arthritis Res Ther 13: 239, 2011 .

59. Allen KD, Adams SB and Setton LA: Evaluating intra-articular drug delivery for the treatment of osteoarthritis in a rat model. Tissue Eng Part B Rev 16: 81-92, 2010.

60. Malfait AM, Little CB and McDougall JJ: A commentary on modelling osteoarthritis pain in small animals. Osteoarthritis Cartilage 21: 1316-1326, 2013.

61. Dequeker J, Hawkey C, Kahan A, Steinbrück K, Alegre C, Baumelou E, Bégaud B, Isomäki H, Littlejohn G, Mau J and Papazoglou S: Improvement in gastrointestinal tolerability of the selective cyclooxygenase (COX)-2 inhibitor, meloxicam, compared with piroxicam: Results of the safety and efficacy large-scale evaluation of COX-inhibiting therapies (SELECT) trial in osteoarthritis. Br J Rheumatol 37: 946-951, 1998. 